\title{
Transmission Protocols in Cognitive Radio Mesh Networks
}

\author{
Anusha M, Srikanth Vemuru, T Gunasekhar \\ Department of Computer science and Engineering, K L University, India
}

\begin{tabular}{|c|c|}
\hline Article Info & ABSTRACT \\
\hline Article history: & A Cognitive Radio (CR) is a radio that can adjust its transmission limit based \\
\hline Received Mar 13, 2015 & Network (CRN) is made up of both the licensed users and unlicensed users \\
\hline Revised Jun 30, 2015 & with CR enable and disabled radios. CR'S supports to access dynamic \\
\hline Accepted Jul 17, 2015 & $\begin{array}{l}\text { spectrum and supports secondary user to access underutilized spectrum } \\
\text { efficiently, which was allocated to primary users. In CRN'S most of the }\end{array}$ \\
\hline Keyword: & $\begin{array}{l}\text { research was done on spectrum allocation, spectrum sensing and spectrum } \\
\text { sharing. In this literature, we present various Medium Access (MAC) }\end{array}$ \\
\hline Cognitive Radios & protocols of CRN'S. This study would provide an excellent study of MAC \\
\hline Licensed users & \\
\hline MAC & \\
\hline Spectrum & \\
\hline Unlicensed users & $\begin{array}{r}\text { Copyright (C) } 2015 \text { Institute of Advanced Engineering and Science. } \\
\text { All rights reserved. }\end{array}$ \\
\hline \multicolumn{2}{|l|}{ Corresponding Author: } \\
\hline $\begin{array}{l}\text { Annusha M, } \\
\text { Research Scholar, } \\
\text { Department of Compute } \\
\text { Greenfields, Vaddeswar } \\
\text { Email: anushaaa9@gma }\end{array}$ & $\begin{array}{l}\text { Engineering, K L University, } \\
\text { istrict, Andhra Pradesh } 522502 .\end{array}$ \\
\hline
\end{tabular}

\section{INTRODUCTION}

A Cognitive Radio (CR) has the capability to sense and collect data, (for example, the communication frequency, transfer speed, and so on) from the adjacent environment [1] and has the ability to adjust rapidly the operational limit, for ideal execution, as indicated by the data sensed [2]. So CR is defined as a radio that can change its transmitter limit according to the communication with the environment in which it works [3]. By using these features we can say that CR technology is the best technology for accessing spectrum dynamically and to effectively utilize underutilized spectrum which was assigned by the federal communications commission (FCC) to authorized users, called as Primary or licensed user. CR'S provide more adaptable and to utilize wide spectrum of restricted and underutilized spectrum [4] for unlicensed users.

CR'S empowers the utilization of unused spectrum transiently which is known as white space or spectrum hole [3], and if licensed user proposes to utilize the same band, then the unlicensed user should move harmlessly to alternative available spectrum or stay in the same band by adjusting its transmission frequency or by modulation approach without interfering the licensed user. When licensed and unlicensed users exist together, protocol spectrum allocation approaches [5] and protocols for spectrum access are not sufficient. If in case, the unlicensed users are permitted to transmit information alongside with licensed user, the transmission ought not to interfere with one another by crossing the edge. In the other case, if the unlicensed user transmitting the information without the licensed user, the unlicensed user should have the ability to identify the reappearance of licensed user and leave the band. There is a lot of exploration as of now being directed and more need to be performed to create new spectrum managing methodologies identified with cognitive radio for both dynamic spectrum sensing and sharing [6-21]. 


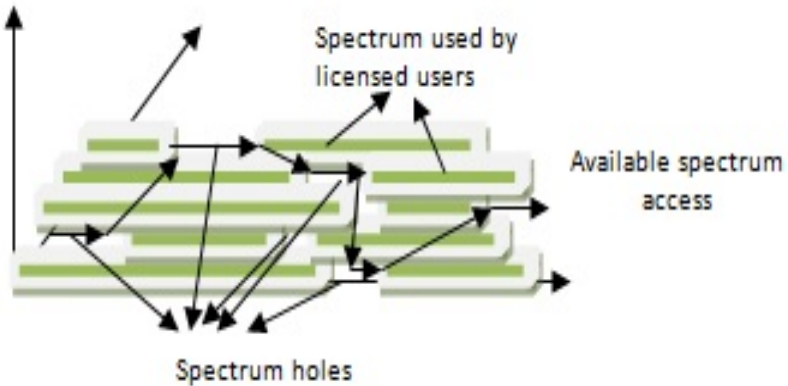

Figure 1. Opportunistic spectrum white space access by unlicensed user

Figure 2 explains the CRN architecture of both licensed users (licensed network) and unlicensed users (unlicensed network). The unlicensed network is a collection of unlicensed users with or without a unlicensed base station, all of which are outfitted with CR capacities. An unlicensed network with a base station is known as Infrastructure based CR Network; the base station acts as a center point to gather the perceptions and consequences of spectrum investigation performed by every CR unlicensed user and make a decision how to reduce interference with licensed user. According to the choice, every CR unlicensed user reconfigures his transmission limit. A unlicensed user without a base station is known as infrastructure less Cognitive Radio Wireless Mesh Network's (CRWMN'S). In a CRWMN'S, the unlicensed users utilize cooperative approach to interchange gathered data among the devices to expand their insight on the whole network, and decides their events. Licensed Network includes licensed users and single or additional base stations, and are not equipped with CR capabilities. Therefore, if unlicensed network imparts a licensed spectrum band with licensed network, the unlicensed network is licensed to be capable to identify the existence of a licensed user and directs the unlicensed transmission to an alternative accessible band that will not interfere with licensed transmission.

Figure 1 explains the opportunistic spectrum white space access and exchanging of frequency bands by unlicensed user at the occurrence of utilization of licensed user. Figure 2 explains the CRN architecture of both licensed network and unlicensed network, with and without infrastructure. The existing spectrum sharing and spectrum allocation approaches as per three conditions [21]: (a) Spectrum bands being used by unlicensed users; (b) network architecture and (c) access behavior of unlicensed users.

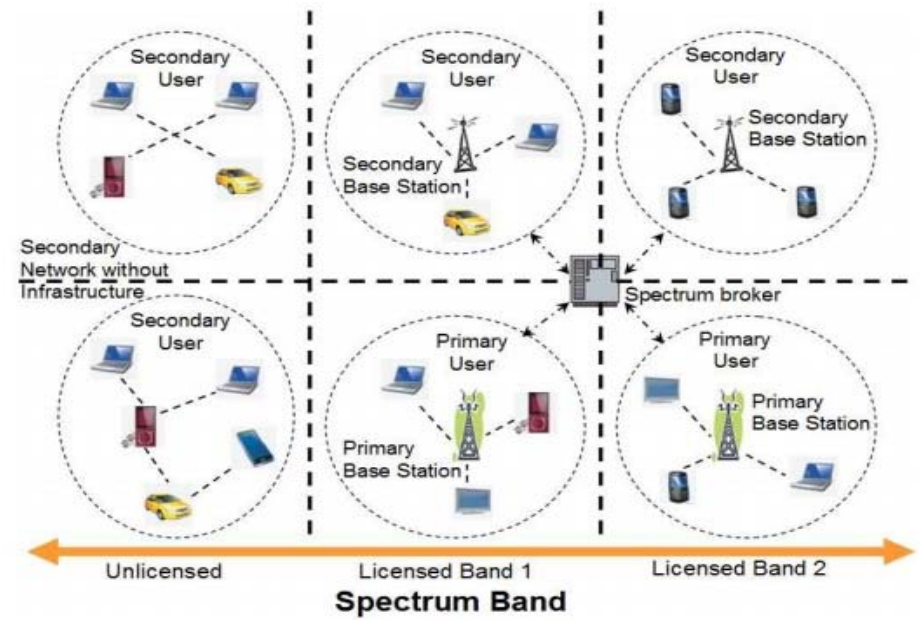

Figure 2. CRN architecture of both licensed network and unlicensed network [21]

\subsection{Utility of Spectrum Bands by Unlicensed User}

According to the spectrum bands used by the unlicensed user, the approaches on sharing of spectrum can be divided as open spectrum sharing and approaches for spectrum access hierarchical. In the approach open spectrum sharing, the unlicensed users get to the unlicensed spectrum and no user possesses any spectrum permit; subsequently, all the users have the same rights to access for utilizing the unlicensed 
spectrum. In the approaches for spectrum access hierarchical [6], the unlicensed users share the licensed spectrum with licensed users. Since licensed users does not require adopting the CR because they have the priority to utilize the spectrum bands. Subsequently, when a licensed user recovers a spectrum band for utilization, the unlicensed user right now utilizing spectrum band and adjacent spectrum bands will need to change their operational limits for avoiding interference with licensed users. The hierarchical spectrum access approach can further divided into two classifications, according to the limitation of unlicensed users:

1) Underlay of spectrum

2) Overlay of spectrum

\subsection{Network Architecture}

According to the network architecture, the spectrum can be partitioned into two approaches - as centralized architecture approach and distributed architecture approach. In centralized approach, a central entity manages and coordinates the allocation of spectrum and access of unlicensed users. In distributed approach, the users take their own decisions according to the spectrum access based on their local examination of dynamic spectrum. The centralized approach is more expensive and further not appropriate for mesh emergency, army services etc., comparatively the distributed approach is less expensive and can be utilized in infrastructure less approach.

\subsection{Access Behaviour of Unlicensed Users}

According to the access behavior of unlicensed users, the spectrum sharing can be classified as either co-operative or non co-operative. In co-operative approach, the unlicensed users often belong to identical service provider and co-ordinate among themselves to enhance the profit to the whole group. In Non Co-Operative approach, the unlicensed users access the open spectrum to enhance the benefits of their own spectrum resources.

\section{COGNITIVE RADIO MAC PROTOCOLS}

In this segment, we are concentrating on the spectrum access issue in which various CR users are distributing the spectrum and decide when and who gets the access to the channel. Here, we are disusing various MAC protocols that have been proposed for both the infrastructure and decentralized CRN'S. The MAC protocols for both classes can be either Random access/Time Division Medium Access (TDMA) or both. The TDMA-based MAC protocols needs network wide synchronization and works by into various time-slots for both the control channel and data transmission. In additional, the Random access protocols does not require time-synchronization, and are based on the principle Carrier Sense Multiple Access/Collision Avoidance (CSMA/CA) in which the secondary user examine the spectrum bands to identify the existence of any other transmission and if so, transmits later backing off for a random span for reducing collisions due to simultaneous transmissions.

\subsection{Centralized MAC Protocols for Cognitive Radio Mesh Networks 2.1.1. Random Access MAC Protocols}

CSMA based random access MAC protocol [7] was proposed for an infrastructure based CRN'S under the supposition of utilization of one transmitter-receiver and in-band signaling. The protocol encourages the co-existence of the licensed user and unlicensed users by adjusting their transmission frequency to preserve the interference to the licensed users inside a predecided limit. The licensed users coordinate with a primary user base station and the unlicensed users coordinate with a secondary user base station, and secure a direct single hop association with their own base stations. The licensed user's takes after the existing CSMA protocol as indicated by which a licensed users senses the channel for a time period (tp) before sending the Request-To-Send (R-T-S) data packet to its base station for which the later may respond with a Clear-To-Send (C-T-S) signal if accessible for the information exchange. The unlicensed users have lot of CS time period (ts, where ts $>>$ tp) so that the licensed users have the priority to access the predecided spectrum. The unlicensed user's base station makes a decision on the transmission power and information rate for exchanging based on the spectrum from itself and unlicensed users. Unlicensed users are permitted to send only one data packet for every transmission to minimize or avoid interference and collisions with licensed users. The random access protocols need an appropriate connection between the licensed and unlicensed networks; generally, the unlicensed users are unaware of any unsuccessful transmissions of licensed users. Additionally, the transmission power of the unlicensed users needs to be separate discrete levels to dependably protect the licensed users from interference and to exploit throughput. 


\subsubsection{Time Division MAC Protocols}

In [8], the Time Division MAC Protocols follows the IEEE 802.22 centralized MAC standard for CRN'S. The 802.22 standard uses basic time slotted multiplexing in the direction of downstream (DS), and requires TDMA (Time Division Multiple Access) in the direction of upstream (US). The base station deals with all the unlicensed users in its cell. Time is divided into different super-frames; A Super frame Control Header ( $\mathrm{SCH}$ ) is positioned at the beginning of every frame to illuminate the unlicensed users about the current accessible channels, various spectrum bandwidths, future spectrum bands access time, and so forth. The MAC frame is made of a DS sub-frame and a US sub-frame. The DS sub-frame takes care of synchronization and channel estimation and a control header contains the sizes of DS-Map and US-Map fields. The DS \& US Map fields have the information about the scheduling of the channels for user convenience. The US sub-frame comprises of a Urgent Coexistence Situation (UCS) notice field that illuminates about the licensed users licensees that have recently been distinguished; alternate fields are utilized to get the separation from the base station and the individual data transmission demands. The primary downside with the time division MAC protocol is the utilization of overwhelming headers as a component of the frames, prompting a decreased throughput.

\subsection{Decentralized MAC Protocols for Cognitive Radio Mesh Networks 2.2.1. Random Access MAC Protocols}

Based on MAC protocol authors proposed [9] a Distributed Channel Assignment (DCA) which uses several transceivers, for signaling which is committed Out-Of-Band control channel and additionally spectrum pooling to dependably recognize the action of the licensed network. Every node preserves a list of channels utilized at that time of its neighbor nodes and list of free channels got from the previous and the spectrum pool. At the period of R-T-S \& C-T-S handshake, the sender and recipient coordinate with their free channels and concur on a same channel to utilize. The R-T-S \& C-T-S messages likewise encourage the neighboring unlicensed users to upgrade their utilized channel and free channel records. The major disadvantage of the DCA protocol is the necessity for a different control channel to support the R-T-S \& CT-S operation, furthermore there is no licensed user related adjustment for channel utilization. In [10], the Single Radio Adaptive Channel (SRAC) algorithm proposed to utilize a FDM model wherein a unlicensed user transmits data packets on a bigger spectrum however gets return affirmations over littler spectrum groups for efficient utilization of spectrum. A CR node keeps list of received spectrum groups of all its neighbor nodes. At the point when a CR node faculties its present transmission channel to be involved by an licensed user, it sends a notice data packet in the received spectrum bands of its neighbor nodes, and changes to the band that is affirmed to by all the neighbor nodes. In the meantime, the CR node transmits on the received spectrum band of a neighboring node that is yet to recognize for the notice data packet. The downside is that signaling the traffic overhead connected with keeping up the upgraded received bands of all the neighbor nodes. Likewise, control messages that are not sent on the receive spectrum groups of a node are not listened to, prompting longer Deaf periods. In [11] \& [12], the CREAM-MAC (Cognitive Radio Enabled Multichannel MAC) and SCA-MAC (Statistical Channel Allocation MAC) protocol are the models of MAC protocols that expect the presence of a worldwide CCC that is approved upon by all the CR nodes in their neighborhood. Under this statement, the working of this class of MAC protocols emulates that of the CSMAstandard for infrastructure networks. While CREAM-MAC is composed in view of a four way handshake process(R-T-S, C-T-S, C-S-T \& C-S-R data packets) on the GCCC, the SCA-MAC utilizes just a two-route dialog process of the control frames (C- R- S \& C- C-S) on the GCCC to support the sender and recipient to tune their transceivers to a commonly settled upon information channel. In [13] \& [14], the Opportunistic Cognitive-MAC (OC-MAC) and the latest Decentralized Non-Global MAC (DNG-MAC) protocols are the samples of MAC protocols that don't require the existence of a worldwide CCC for choosing spectrum access among neighboring unlicensed users. OC-MAC expect that the CRN exists together with a WLAN and utilization of the IEEE 802.11DCF (Distributed Coordination function) method at the CR nodes to rival each other for information channel reservation. The DNG-MAC protocol utilizes the TDMA to allocate the control channel to all the accessible CR nodes; the CCC is one of the best accessible channels chose by the first CR node that starts the information correspondence. The CCC is partitioned into time-slots of static length; every time-slot containing a listening period and a transceiving period. The reason of DNG-MAC is that since all CR nodes starve for a data channel to utilize, there won't be wastage of the assets with the task of a period space of the control channel for each CR node. In spite of this assumption improves the model of DNG-MAC and stays away from the complex synchronization overhead normally seen with time-opening based MAC protocols, it is complex to suppose the information channels to be accessible for the same time span as that of the time-slots of the control channel and the time-slot every CR node must be re-computed upon the consideration/rejection of a CR node in the network. This likewise suggests that the MAC protocol to be additionally not adaptable for changes in the network topology because of node versatility. 


\subsubsection{Time Division MAC Protocols}

We examine the C-MAC [15] supports synchronized time divisions by including the utilization of a Rendezvous channel (Rc) and a Backup channel (Bc). The Rc exists maximum time for utilization for unlicensed users all over the network and is utilized for node coordination, licensed user identification, and also multi channel asset reservation. The Bc is mainly decided at every unlicensed users, all through of-band estimations, and is utilized as alternative spectrum on account of appearance of an unlicensed users. In CMAC protocol, every spectrum includes recurring of super-frames. Every super-frame is made up of a Beacon Period (BP) and a Data Transmission Period (D-T-P). Every BP is a time division so that the individual unlicensed user can transmit their signals without interference. The Rc is utilized to switch the BP schedules of nodes to prevent concurrent transmission over all the spectrum. An Unlicensed user declares the requirement for any new spectrum information through the beacons, and likewise illuminates about any spectrum change over the Rc. Occasional tuning to the Rc permits unlicensed user to re-synchronize and acquire the latest neighborhood topology data. The time division nature of C-MAC encourages the utilization of a non overlapping Quiet Period (QP) for every spectrum, through which one could separate licensed user from unlicensed user. The major disadvantages of the C-MAC are that it requires the Rc to be a committed spectrum that is not utilized by any primary user, which is hard to ensure in decentralized networks. Additionally, because of the requirement to incorporate the beacon signals with the load and channel utilization data in the BP of a super-frame, the protocol is not adaptable for a large number of unlicensed users. It is complex to support the non-overlapping nature of the BP's and the quiet periods, without the existence of a central element. A distributed time division protocol [16] was proposed to avoid the utilization of Rc by giving in-band signaling through a committed control window in addition to the beacon signal and information exchange periods.

\section{CONCLUSION}

In this study, we have presented an extensive study and analysis of cognitive radio networks MAC protocols basec on centralized and decentralized networks and existing solutions. From this point of view, primary user need not be aware of the secondary users, and there should be no significant stage in the quality of service for the licensed users. While the results proposed for centralized and decentralized CRNs are regularly interpreted to give execution benchmarks to the suitable model, the results proposed for distributed or co-operative and decentralized mesh CRN'S and execute bottlenecks in real time executions. Majority of research done in the area of CRN'S focused on spectrum sensing, spectrum allocation and spectrum sharing and MAC.

\section{REFERENCES}

[1] FCC, ET Docket No 02-135, Spectrum Policy Taskforce Report, 2002.

[2] S. Haykin, "Cognitive radio: Brain-empowered wireless communications", IEEE Journal of Selected Areas in Communications, Vol. 23, No. 2, pp. 201-220, 2005.

[3] FCC, ET Docket No 03-222, Notice of Proposed Rule Making and Order, 2003.

[4] I. F. Akyildiz, W. Y. Lee, M. C. Vuran, and S. Mohanty, "Next Generation/Dynamic Spectrum Access/Cognitive Radio Wireless Networks: A Survey”, Computer Networks, Vol. 50, pp. 2127-2159, 2006.

[5] Z. Han, and K. J. R. Liu, "Resource Allocation for Wireless Networks: Basics”, Techniques and Applications, Cambridge University Press, Cambridge, UK, 2008.

[6] Q. Zhao, and B. Sadler, “A survey of Dynamic Spectrum Access”, IEEE Signal Processing Magazine, Vol. 24, No. 3, pp. 79-89, 2007.

[7] S. Y. Lien, C. C. Tseng, and K. C. Chen, "Carrier Sensing based Multiple Access Protocols for Cognitive Radio Networks”, Proceedings of IEEE International Conference on Communications, pp. 3208-3214, 2008.

[8] C. Cordeiro, K. Challapali, and M. Ghosh, "Cognitive PHY and MAC Layers for Dynamic Spectrum Acess and Sharing of TV Bands”, Proceedings of IEEE International Workshop on Technology and Policy for Accessing Spectrum, pp. 222, 2006.

[9] P. Pawelczak, R. Venkatesha Prasad, L. Xia, and I. G. M. M. Niemegeers, "Cognitive Radio Emergency NetworksRequirements and Design”, Proceedings of the IEEE Dynamic Spectrum Access Networks, pp. 601-606, 2005.

[10] L. Ma, C. C. Shen, and B. Ryu, "Single Radio Adaptive Channel Algorithm for Spectrum Agile |Wireless Ad hoc Networks”, Proceedings of the IEEE Dynamic Spectrum Access Networks, pp. 547-558, 2007.

[11] H. Su, "CREAM-MAC: An Efficient Cognitive Radio enabled Multi-Channel MAC Protocol for wireless Networks", Proceedings of the International Symposium on World of Wireless, Mobile and Mulimedia Networks, 2008.

[12] Y. R. Kondareddy, and P. Agrawal, "Synchronized MAC Protocol for Multi-hop Cognitive Radio Networks", Proceedings of the IEEE International Conference on Communications, pp. 3198-3202, 2008. 
[13] S. Y. Hung, E. H. K. Wu, and G. H. Chen, "An Opportunistic Cognitive MAC Protocol for Coexistence with WLAN”, Proceedings of the IEEE International Conference on Communication, pp. 4059-4063, 2008.

[14] M. A. Shah, S. Zhang, and C. Maple, "An Analysis on Decentralized Adaptive MAC Protocols for Cognitive Radio Networks", Proceedings of the 18 "International Conference on Automation and Computing, 2012.

[15] C. Cordeiro and K. Challapali, "C-MAC: A Cognitive MAC Protocol for Multichannel Wireless Networks", Proceedings of the IEEE Dynamic Spectrum and Access Networks, pp. 147-157, 2007.

[16] J. Zhao, H. Zheng, and G. H. Yang, "Spectrum Sharing through Distributed Coordination in Dynamic Spectrum Access Networks”, Wireless Communications and Mobile Computing, Vol. 7, No. 9, pp. 1061-1075, 2007.

[17] Z. Htike, J. Lee, and C. S. Hong, "A MAC Protocol for Cognitive Radio Networks with Reliable Control Channels Assignment”, Proceedings of the International Conference on Information Networking, pp. 81-85, 2012.

[18] L. Dasilva and I. Guerreiro, "Sequence based Rendezvous for Dynamic Spectrum Access", Proceedings of the IEEE Conference on Dynamic Spectrum Access Networks, 2008.

[19] K. Bian, J. M. Park, and R. Chen, ”A Quorum based Framework for establishing Control Channels in Dynamic Spectrum Access Networks", Proceedings of the ACM International Conference on Mobile Computing (MobiCom), pp. 25-36, 2009.

[20] Y. H. Lee and D. Kim, “A Slow Hopping MAC Protocol for Coordinator based Cognitive Radio Network”, Proceedings of the IEEE Consumer Communications and Networking Conference, pp. 854-858, 2012.

[21] Natarajan Meghananthan, "A survey on the Communication Protocols and Security in Cognitive Radio Networks", International Journal of Communication Networks and Information Security (IJCNIS), Vol. 5, pp. 19-38, 2013. 